\title{
Applications of Some Topological Near Open Sets to Knowledge Discovery
}

\author{
A. S. Salama \\ Tanta University; Shaqra University \\ Tanta, Egypt; Sajir, KSA
}

\author{
O. G. El-Barbary \\ Shaqra University \\ Faculty of Science, Tanta University \\ Sajir, KSA
}

\begin{abstract}
In this paper, we use some topological near open sets to introduce the rough set concepts such as near open lower and near open upper approximations. Also, we study the concept of near open, rough set and some of their basic properties. We will study the comparison among near open concepts and rough set concepts. We also studied the effect of this concept to motivate the knowledge discovery processing.
\end{abstract}

Keywords-Topological spaces; Rough sets; Knowledge discovery; open sets; Accuracy measure

\section{INTRODUCTION}

Information technology is huge and need more accurate measures to discover their valuable knwoledge. An important field of this technology is the information discovery via available information. Rough set theory and their topological generalizations using some topological near open sets $[1,2,4$, $5,7,12,13,15,17,18,19,20]$ are a recent, an accurate and applicable approach for reasoning about data. Many researchers generalized rough sets, but the concept of a topological rough set given by Wiweger [16] in 1989 is the basic starting point. This generalization is basically starting with a topological space and defined the approximation via the interior and the closure operators on topological spaces. The concept of $\beta$-open set was introduced in 1983 by M. E. Abd El-Monsef and others [3].

In this paper, we introduce and investigate the concept of near open approximation space. These spaces help to get a new classification for the universe. Also, we investigate the concept of near open lower and near open upper approximations. We study near open, rough sets, the comparison between this concept and rough sets is also studied. Also, we give some counter examples.

\section{ROUGH SET BASIC CONCEPTS}

Classical rough set theory has come from the need to represent subsets of a universe in terms of equivalence classes of a partition of that universe. The partition characterizes a topological space, called approximation space $K=(X, R)$, where $X$ is a set called the universe and $R$ is an equivalence relation $[8,14]$. The equivalence classes of $R$ are also known as the granules, elementary sets or blocks; we will use $R_{x} \subseteq X$ to denote the equivalence class containing $x \in X$. In the approximation space, we consider two sets, namely
$\bar{R}(A)=\left\{x \in X: R_{x} \cap A \neq \phi\right\}$

and $\underline{R}(A)=\left\{x \in X: R_{x} \subseteq A\right\}$ that is called the lower and the upper approximation of $A \subseteq X$ respectively. Also, let $\operatorname{POS}_{R}(A)=\underline{R}(A)$ denote the positive region of $A$, $N E G_{R}(A)=X-\bar{R}(A)$ denotes the negative region of $A$ and $B N_{R}(A)=\bar{R}(A)-\underline{R}(A)$ denote the boundary region of $\mathrm{X}$.

The degree of completeness can also be characterized by the accuracy measure, in which $|R|$ represents the cardinality of set $R$ as follows:

$$
\alpha_{R}(A)=\frac{|\underline{R}(A)|}{|\bar{R}(A)|} \text { where } A \neq \phi .
$$

Accuracy measures try to express the degree of completeness of knowledge. $\alpha_{R}(A)$ is able to imprisonment, how large the boundary region of the data sets is; however, we cannot easily capture the structure of the knowledge. A fundamental advantage of rough set theory is the ability to handle a category that cannot be sharply defined given a knowledge base. Characteristics of the potential data sets can be measured through the rough sets framework. We can measure inexactness and express topological characterization of imprecision with:

(1) If $\underline{R}(A) \neq \phi$ and $\bar{R}(A) \neq X$, then $A$ is roughly $R$ definable.

(2) If $\underline{R}(A)=\phi$ and $\bar{R}(A) \neq X$, then $A$ is internally $R$ undefinable

(3) If $\underline{R}(A) \neq \phi$ and $\bar{R}(A)=X$, then $A$ is externally $R$ undefinable.

(4) If $\underline{R}(A)=\phi$ and $\bar{R}(A)=X$, then $A$ is totally $R$ undefinable.

We denote the set of all, roughly $R$-definable (resp. Internally $R$-undefinable, externally $R$-undefinable and totally $R$-undefinable) sets by $R D(X)$ (resp. $\operatorname{IUD}(X)$, $\operatorname{EUD}(X)$ and $T U D(X))$. 
With $\alpha_{R}(A)$ and classifications above, we can characterize rough sets by their size of the boundary region.

A topological space [6] is a pair $(X, \tau)$ consisting of a set $X$ and family $\tau$ of subsets of $X$ satisfying the following conditions:
(1) $\phi, X \in \tau$
(2) $\tau$ is closed under arbitrary union.
(3) $\tau$ is closed under finite intersection.

The pair $(X, \tau)$ is called a topological space, the elements of $X$ are called points of the space, the subsets of $X$ belonging to are called open set in the space, and the complement of the subsets of $X$ belonging, to $\tau$ are called closed set in the space; the family $\tau$ of open subsets of $X$ is also called a topology for $X$.

$$
\bar{A}=\bigcap\{F \subseteq X: A \subseteq F \text { and } F \text { is closed }\} \text { is }
$$

called $\tau$-closure of a subset $A \subset X$.

Evidently, $A$ is the smallest closed subset of $X$ which contains $A$. Note that A is closed iff $A=\bar{A}$.

$$
A^{\circ}=\bigcup\{G \subseteq X: G \subseteq A \text { and } G \text { is open }\} \text { is }
$$

called the $\tau$-interior of a subset $A \subseteq X$.

Evidently, $A^{\circ}$ is the union of all open subsets of $X$ which containing in $A$. Note that $\mathrm{A}$ is open iff $A=A^{\circ}$. And $b(A)=\bar{A}-A^{\circ}$ is called the $\tau$-boundary of a subset $A \subseteq X$.

Let $A$ be a subset of a topological space $(X, \tau)$. Let $\bar{A}$, $A^{\circ}$ and $b(A)$ be closure, interior, and boundary of $A$ respectively. $A$ is exact if $b(A)=\phi$, otherwise $A$ is rough. It is clear $A$ is exact iff $\bar{A}=A^{\circ}$. In Pawlak space a subset $A \subseteq X$ has two possibilities rough or exact.

Definition 2.1. [3] A subset $A$ of a topological space $(X, \tau)$ is called $\beta$-open

$$
\text { if } A \subset \operatorname{cl}(\operatorname{int}(\operatorname{cl}(A))) \text {. }
$$

The complement of $\beta$-open set is $\beta$-closed set. We denote that the set of all $\beta$ - open and $\beta$-closed sets by $\beta O(X)$ and $\beta C(X)$ respectively.

Remark 2.1. For any topological space $(X, \tau)$. We have $\tau \subseteq \beta O(X)$.

\section{Topological Near Open Rough Classification}

In this section, we introduce and investigate the concept of near open approximation space. Also, we introduce the concepts of near open lower approximation and near open upper approximation and study their properties.

Definition 3.1. Let $X$ be a finite non-empty universe. The pair $\left(X, R_{\beta}\right)$ is called a near open approximation space where $R_{\beta}$ is a general relation used to get a subbase for a topology $\tau$ on $X$ and a class of $\beta$-open sets $\beta O(X)$.

Remark 3.1. In Definition 3.1, we use the symbol $R_{\beta}$ to avoid confusion with $R$ which refers to an equivalence relation.

Example 3.1. Let $U=\{a, b, c, d, e, f, g\}$ be a universe of multi valued information system of seven patients (A, B and $\mathrm{C}$ are conditional attributes and $\mathrm{D}$ is the decision attribute) as in Table 1 , the relation $R$ defined on patients by $R=\{(a, a), \quad(a, c),(a, d),(b, b),(b, d), \quad(c, a),(c, b)$, $(c, d),(d, a)\}$, thus $a R=\{a, c, d\}, b R=\{b, d\}$, $c R=\{a, b, d\}$ and $d R=\{a\}$. Then the topology associated with this relation is $\tau=\{U, \phi,\{a\}$, $\{d\},\{a, d\}, \quad\{b, d\}, \quad\{a, b, d\}, \quad\{a, c, d\}\} \quad$ and $\beta O(X)=\{X, \quad \phi,\{a\}, \quad\{d\},\{a, c\}, \quad\{a, d\}$, $\{b, d\},\{c, d\},\{a, b, d\},\{a, c, d\},\{b, c, d\}\}$. So $\left(U, R_{\beta}\right)$ is a near open approximation space.

TABLE I. Multivalued INFORMATION System

\begin{tabular}{|l|l|l|l|l|}
\hline U & A & B & C & D \\
\hline a & $\{$ A2 $\}$ & $\{$ B1,B2,B4 $\}$ & $\{\mathrm{c} 1\}$ & $\{$ d3 $\}$ \\
\hline b & $\{$ A1,A2 $\}$ & $\{$ B1,B2 $\}$ & $\{\mathrm{c} 1, \mathrm{c} 3\}$ & $\{\mathrm{d} 3\}$ \\
\hline c & $\{\mathrm{A} 2\}$ & $\{\mathrm{B} 1, \mathrm{~B} 2\}$ & $\{\mathrm{c} 1\}$ & $\{\mathrm{d} 3\}$ \\
\hline d & $\{\mathrm{A} 1\}$ & $\{\mathrm{B} 1, \mathrm{~B} 2, \mathrm{~B} 4\}$ & $\{\mathrm{c} 4\}$ & $\{\mathrm{d} 1\}$ \\
\hline e & $\{\mathrm{A} 1\}$ & $\{\mathrm{B} 5\}$ & $\{\mathrm{c} 1, \mathrm{c} 2\}$ & $\{\mathrm{d} 2\}$ \\
\hline f & $\{\mathrm{A} 1\}$ & $\{\mathrm{B} 1, \mathrm{~B} 2\}$ & $\{\mathrm{c} 1\}$ & $\{\mathrm{d} 3\}$ \\
\hline g & $\{\mathrm{A} 1\}$ & $\{\mathrm{B} 1, \mathrm{~B} 3, \mathrm{~B} 4\}$ & $\{\mathrm{c} 1, \mathrm{c} 3\}$ & $\{\mathrm{d} 3\}$ \\
\hline
\end{tabular}

Example 3.2. Let $X=\{a, b, c\}$ be a subset of the universe $U$, and a relation $R$ defined on $X$ by $a R=\{a, b\}, \quad b R=\{b\}, \quad c R=\{a, b\}$. Then the subtopology associated with this relation is $\tau=\{X, \phi$, $\{b\},\{a, b\}\}$ and $\beta O(X)=\{X, \phi,\{b\},\{a, b\},\{b, c\}\}$. So $\left(X, R_{\beta}\right)$ is a subnear open approximation space.

Definition 3.2. Let $\left(X, R_{\beta}\right)$ be a near open approximation space near open lower (resp. near open upper) approximation of any non-empty subset $A$ of $X$ is defined as:

$$
\begin{aligned}
& \underline{R}_{\beta}(A)=\bigcup\{G \in \beta O(X): G \subseteq A\} \\
& \bar{R}_{\beta}(A)=\bigcap\{F \in \beta C(X): F \supseteq A\}
\end{aligned}
$$

Definition 3.3. Let $\left(X, R_{\beta}\right)$ be a near open approximation space. From the relation 
$\operatorname{int}(A) \subseteq \beta \operatorname{int}(A) \subseteq A \subseteq \beta \operatorname{cl}(A) \subseteq \operatorname{cl}(A) \quad$, for any $A \subseteq X$. The Universe $X$ can be divided into 12 regions with respect to any $A \subseteq X$ as shown in Figure 1 .

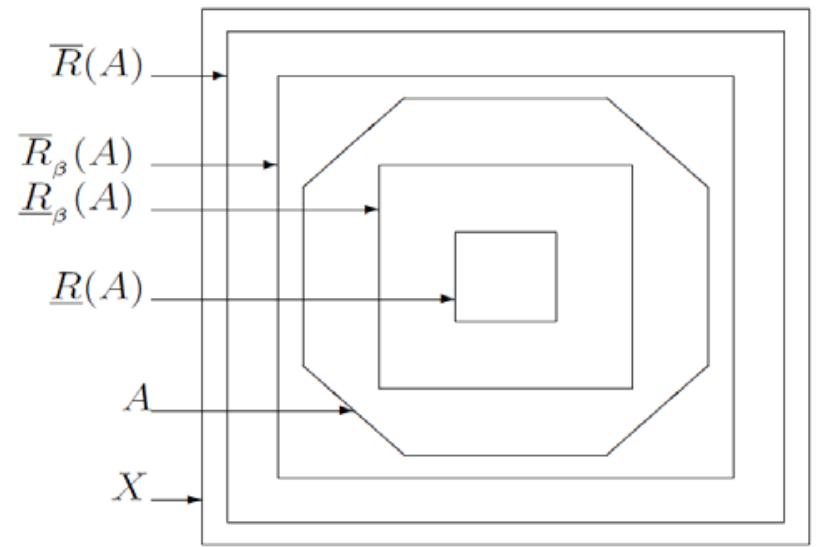

Fig. 1. Regions of the universe

Remark 3.2. The study of near open approximation spaces is a generalization for the study of approximation spaces. Because of the elements of the regions $\left[\underline{R}_{\beta}(A)-\underline{R}(A)\right]$ will be defined well in $A$, while this point was undefinable in Pawlak's approximation spaces. Also, the elements of the region $\left[\bar{R}(A)-\bar{R}_{\beta}(A)\right]$ do not belong to $A$, while these elements was not well defined in Pawlak's approximation spaces.

In our study, reduce the boundary of $A$, in Pawlak's approximation space by near open boundary of $A$. Also, we extend exterior of $A$ which contains the elements did not belong to $A$ by near open exterior of $A$.

Proposition 3.1. For any near open approximation space $\left(X, R_{\beta}\right)$, the following are hold of any $A \subseteq X$ :

(1) $b(A)=\underline{E d g}(A) \cup \overline{E d g}(A), \underline{E d g}(A)=A-\underline{R}(A)$,

$\overline{E d g}(A)=\bar{R}(A)-A$

(2) $\beta b(A)=\beta E d g(A) \cup \beta \overline{E d g}(A)$,

$\beta E \operatorname{Edg}(A)=A-\underline{R}_{\beta}(A), \beta \overline{E d g}(A)=\bar{R}_{\beta}(A)-A$

Proof (2). It follows from

$$
\begin{aligned}
\beta b(A)= & \bar{R}_{\beta}(A)-\underline{R}_{\beta}(A) \\
& =\left(\bar{R}_{\beta}(A)-A\right) \cup\left(A-\underline{R}_{\beta}(A)\right) \\
& =\beta \underline{E d g}(A) \cup \beta \overline{\operatorname{Edg}}(A)
\end{aligned}
$$

Proposition 3.2. For any near open approximation space ( $X, R_{\beta}$ ), the following are hold of any $A \subseteq X$ :

(1) $\bar{R}(A)-\underline{R}_{\beta}(A)=\overline{E d g}(A) \cup \beta \underline{E d g}(A)$
(2) $\bar{R}_{\beta}(A)-\underline{R}(A)=\beta \overline{E d g}(A) \cup \underline{E d g}(A)$

Proof. Obvious.

Proposition 3.3. For any near open approximation space $\left(X, R_{\beta}\right)$. The following are hold to any $A \subseteq X$ :

(1) $\underline{E d g}(A)=\beta \underline{E d g}(A) \cup\left(\underline{R}_{\beta}(A)-\underline{R}(A)\right)$

(2) $\overline{E d g}(A)=\beta \overline{E d g}(A) \cup\left(\bar{R}(A)-\bar{R}_{\beta}(A)\right)$

Proof. Obvious.

Definition 3.4. Let $\left(X, R_{\beta}\right)$ be a near open approximation space and $A \subseteq X$. Then there are memberships $\in, \bar{\epsilon}, \in_{\beta}$ and $\bar{\epsilon}_{\beta}$, say, near open strong and near open weak memberships respectively which defined by:

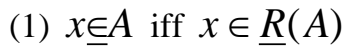

(2) $x \bar{\in} A$ iff $x \in \bar{R}(A)$

(3) $x \underline{\in}_{\beta} A$ iff $x \in \underline{R}_{\beta}(A)$

(4) $x \bar{\in}_{\beta} A$ iff $x \in \bar{R}_{\beta}(A)$

Remark 3.3. According to Definition 3.4, near open lower and near open upper approximations of a set $A \subseteq X$ can be written as

(1) $\underline{R}_{\beta}(A)=\left\{x \in A: x \underline{\epsilon}_{\beta} A\right\}$

(2) $\bar{R}_{\beta}(A)=\left\{x \in A: x \bar{x}_{\beta} A\right\}$

Remark 3.4. Let $\left(X, R_{\beta}\right)$ be a near open approximation space and $A \subseteq X$. Then

(1) $x \in A \Rightarrow x \in \in_{\beta} A$

(2) $x \bar{\in}_{\beta} A \Rightarrow x \bar{\in} A$

The converse of Remark 3.4 may not be true in general as seen in the following example.

Example 3.3. Let $X=\{a, b, c, d\}$ be a universe and a relation $R$ defined by $R=\{(a, a),(d, c),(d, d)$, $(c, a),(c, d),(c, c)\}$, thus $a R=\{a\}, b R=\phi$, $c R=\{a, c, d\}$ and $d R=\{c, d\}$. Then the topology associated with this relation is $\tau=\{X, \phi,\{a\}$, $\{c, d\},\{a, c, d\}\}$. So $\left(X, R_{\beta}\right)$ is a $\beta$-approximation space. Let, $A=\{b, c, d\}$, we have $b \underline{\in}_{\beta} A$ but $b \underline{\notin}$. Also, let $B=\{c\}$. We have $d \quad \bar{\in} B$ but, $d \bar{\notin}_{\beta} B$.

We can characterize the degree of completeness by a new tool named $\beta$-accuracy measure defined as follows: 


$$
\alpha_{R_{\beta}}(A)=\frac{\left|\underline{R}_{\beta}(A)\right|}{\left|\bar{R}_{\beta}(A)\right|} \text { where } A \neq \phi
$$

Example 3.4. According to Example 3.1, we can deduce Table 2 below that show the degree of accuracy measure $\alpha_{\beta}(A)$ and $\beta$-accuracy measure $\alpha_{R_{\beta}}(A)$ for some proper subsets of the universe of discourse.

TABLE II. ACCURACY MEASURES

\begin{tabular}{|l|l|l|}
\hline$A \subseteq X$ & $\alpha_{\beta}(A)$ & $\alpha_{R_{\beta}}(A)$ \\
\hline$\{\mathrm{a}\}$ & $1 / 2$ & 1 \\
\hline$\{\mathrm{a}, \mathrm{c}\}$ & $1 / 2$ & 1 \\
\hline$\{\mathrm{b}, \mathrm{d}\}$ & $1 / 3$ & 1 \\
\hline$\{\mathrm{b}, \mathrm{c}, \mathrm{d}\}$ & $2 / 3$ & 1 \\
\hline
\end{tabular}

We see that the degree of exactness of the set $A=\{a\}$ by using accuracy measure equal to $50 \%$ and by using near open accuracy measure equal to $100 \%$. Consequently near open accuracy measure is the accurate measure in this case.

We investigate near open, rough equality and near open rough inclusion based on rough equality and inclusion which introduced by Pawlak and Novotny in $[10,11]$.

Definition 3.5. Let $\left(X, R_{\beta}\right)$ be a near open approximation space, $A, B \subseteq X$. Then we say that $A$ and $B$ are:

(i) Near open roughly bottom equal if $\underline{R}_{\beta}(A)=\underline{R}_{\beta}(B)$

(ii) Near open roughly top equal if $\bar{R}_{\beta}(A)=\bar{R}_{\beta}(B)$

(iii) Near open roughly equal $\left(A \approx_{\beta} B\right)$ if

$\underline{R}_{\beta}(A)=\underline{R}_{\beta}(B)$ and $\bar{R}_{\beta}(A)=\bar{R}_{\beta}(B)$

Example 3.5. According to Example 3.1, we have the sets $\{a, c\},\{a, b, c\}$ are near open roughly bottom equal and $\{c, d\},\{b, c, d\}$ are near open roughly top equal.

One can easily show that $\approx_{\beta}$ is an equivalence relation on $P(X)$, hence the pair $\left(P(X), \approx_{\beta}\right)$ is an approximation space. Also, this relation $\approx_{\beta}$ is called near open, rough equality of the near open approximation space $\left(X, R_{\beta}\right)$.

Definition 3.6. Let $\left(X, R_{\beta}\right)$ be a near open approximation space. We define the equivalence relation $E_{\beta}$ on the set $P(X)$ given by the condition:

$$
(A, B) \in E_{\beta} \quad \text { if } \quad \beta \quad-\quad \operatorname{int}(A)=\beta \quad-
$$

$\operatorname{int}(B) \quad$ and $\quad \beta-\operatorname{cl}(A)=\beta-\operatorname{cl}(B)$.

The equivalence relation $E_{\beta}$ is precisely the same of $\approx_{\beta}$, since $\underline{R}_{\beta}(A)=\beta \operatorname{int}(A)$ and $\bar{R}_{\beta}(A)=\beta \operatorname{cl}(A)$.

Remark 3.5. For any subset $A$ of $X$, the equivalence class of the relation $\left(\approx_{\beta}\right.$ or $E_{\beta}$ ) containing $A$ is denoted by $[A]_{\approx_{\beta}}$ or $[A]_{E_{B}}$. Thus,

$$
\begin{gathered}
{[A]_{\approx_{\beta}}=\left\{D \subset X: \underline{R}_{\beta}(D)=\underline{R}_{\beta}(A)\right. \text { and }} \\
\left.\bar{R}_{\beta}(D)=\bar{R}_{\beta}(A)\right\} .
\end{gathered}
$$

We denote by $R_{\beta}(X)$ of the family of near open rough classes of a near open approximation space $\left(X, R_{\beta}\right)$.

Definition 3.7. Let $\left(X, R_{\beta}\right)$ be a near open approximation space, $A, B \subseteq X$. Then we say that

(i) $A$ is near open roughly bottom included in $B$ if $\underline{R}_{\beta}(A) \subseteq \underline{R}_{\beta}(B)$

(ii) $A$ is near open roughly top included in $B$ if $\bar{R}_{\beta}(A) \subseteq \bar{R}_{\beta}(B)$

(iii) $A$ is near open roughly included in $B$ if $\underline{R}_{\beta}(A) \subseteq \underline{R}_{\beta}(B)$ and $\bar{R}_{\beta}(A) \subseteq \bar{R}_{\beta}(B)$

Example 3.6. According to Example 3.1, we have $\{a, c\}$ is near open roughly bottom included in $\{a, b, c\}$. Also, $\{c, d\}$ is near open roughly top included in $\{b, c, d\}$.

\section{Properties of Near Open, Rough Sets}

In this section, we introduced a new concept of near open rough set.

Definition 4.1. For any near open approximation space $\left(X, R_{\beta}\right)$, a subset $A$ of $X$ is called:

(1) $\quad R_{\beta}$-definable $\left(\beta\right.$-exact)if $\bar{R}_{\beta}(A)=\underline{R}_{\beta}(A)$ or $\beta b(A)=\phi$

(2) near open rough if $\bar{R}_{\beta}(A) \neq \underline{R}_{\beta}(A)$ or $\beta b(A) \neq \phi$

Example 4.1. Let $\left(X, R_{\beta}\right)$ be a near open approximation space as in Example 3.3. We have the set $\{a, c\}$ is near open exact while $\{b\}$ is near open rough set.

Proposition 4.1. Let $\left(X, R_{\beta}\right)$ be a near open approximation space. Then:

(1) Every exact set in $X$ is near open exact.

(2) Every near open rough set in $X$ is rough.

Proof. Obvious.

The converse of all parts of Proposition 4.2 may not be true in general as seen in the following example. 
Example 4.2. Let $\left(X, R_{\beta}\right)$ be a near open approximation space as in Example 3.3. Then the set $\{a, c\}$ is near open exact, but not exact and the set $\{a, b, d\}$ is rough but not near open rough.

Remark 4.1. The intersection of two near open exact sets need not be near open exact set.

Example 4.3. Let $\left(X, R_{\beta}\right)$ be a near open approximation space as in Example 3.3. We have $\{b, c\}$ and $\{a, b\}$ are two near open exact sets but $\{b, c\} \cap\{a, b\}=\{b\}$ does not near open exact.

Definition 4.2. Let $\left(X, R_{\beta}\right)$ be a near open approximation space, the set $A \subseteq X$ is called:

(1) Roughly $R_{\beta}$-definable, if $\underline{R}_{\beta}(A) \neq \phi$ and $\bar{R}_{\beta}(A) \neq X$.

(2) Internally $R_{\beta}$-undefinable, if $\underline{R}_{\beta}(A)=\phi$ and

$\bar{R}_{\beta}(A) \neq X$.

(3) Externally $R_{\beta}$-undefinable, if $\underline{R}_{\beta}(A) \neq \phi$ and

$\bar{R}_{\beta}(A)=X$.

(4) Totally $R_{\beta}$-undefinable, if $\underline{R}_{\beta}(A)=\phi$ and

$\bar{R}_{\beta}(A)=X$.

We denote the set of all, roughly $R_{\beta}$-definable (resp. Internally $R_{\beta}$-undefinable, externally $R_{\beta}$-undefinable and totally $\quad R_{\beta}$-undefinable) sets by $\beta R D(X) \quad$ (resp. $\beta \operatorname{IUD}(X), \beta E U D(X)$ and $\beta T U D(X))$.

Remark 4.2. For any near open approximation space $\left(X, R_{\beta}\right)$. The following are held:

(1) $\beta R D(X) \supseteq R D(X)$

(2) $\beta I U D(X) \subseteq I U D(X)$

(3) $\beta E U D(X) \subseteq E U D(X)$

(4) $\beta T U D(X) \subseteq T U D(X)$

Example 4.4. According to Example 3.3, we have the set $\{a, b\} \in \beta R D(X)$ but $\{a, b\} \notin R D(X)$. The set $\{b, d\} \in I U D(X)$ but $\{b, d\} \notin \beta I U D(X)$. Also, the set $\{a, d\} \in E U D(X)$ but $\{a, d\} \in \beta E U D(X)$.

Lemma 4.1. For any near open approximation space $\left(X, R_{\beta}\right)$ and for all $x, y \in X$, the condition $x \in \bar{R}_{\beta}(\{y\})$ and $y \in \bar{R}_{\beta}(\{x\})$ implies $\bar{R}_{\beta}(\{x\})=\bar{R}_{\beta}(\{y\})$.

Proof. By definition of near open upper approximation of a set is a $\beta$-closure of this set, and since $\beta \operatorname{cl}(\{y\})$ is a $\beta$ closed set containing $x$ (by the condition) while $\beta c l(\{x\})$ is the smallest $\beta$-closed set containing $X$, thus $\beta c l(\{x\}) \subseteq \beta \operatorname{cl}(\{y\})$. Hence $\bar{R}_{\beta}(\{x\}) \subseteq \bar{R}_{\beta}(\{y\})$. The opposite inclusion follows by symmetry $\beta c l(\{y\}) \subseteq \beta \operatorname{cl}(\{x\})$. Hence $\bar{R}_{\beta}(\{y\}) \subseteq \bar{R}_{\beta}(\{x\})$, which complete the proof.

Lemma 4.2. Let $\left(X, R_{\beta}\right)$ be a near open approximation space, which every $\beta$-open subset $A$ of $X$ is $\beta$-closed. Then $y \in \bar{R}_{\beta}(\{x\})$ implies $x \in \bar{R}_{\beta}(\{y\})$ for all $x, y \in X$.

Proof. If $x \notin \bar{R}_{\beta}(\{y\})$, then there exists a $\beta$-open set $G$ containing $x$ such that $G \cap\{y\}=\phi$ which implies that $\{y\} \subseteq(X \backslash G)$, but $(X \backslash G)$ is a $\beta$-closed set and also is a $\beta$-open set does not contain $x$, thus $(X \backslash G) \cap\{x\}=\phi$. Hence $y \notin \bar{R}_{\beta}(\{x\})$.

Proposition 4.2. Let $\left(X, R_{\beta}\right)$ be a near open approximation space, and every $\beta$-open subset $A$ of $X$ is $\beta$-closed. Then the family of sets $\left\{\bar{R}_{\beta}(\{x\}): x \in A\right\}$ is a partition of the set $X$.

Proof. If $x, y, z \in A$ and $z \in \bar{R}_{\beta}(\{x\}) \cap \bar{R}_{\beta}(\{y\})$, then $z \in \bar{R}_{\beta}(\{x\})$ and $z \in \bar{R}_{\beta}(\{y\})$. Thus by Lemma 4.2, $x \in \bar{R}_{\beta}(\{z\})$ and $y \in \bar{R}_{\beta}(\{z\})$ and by Lemma 4.1 we have $\bar{R}_{\beta}(\{x\})=\bar{R}_{\beta}(\{z\})$ and $\bar{R}_{\beta}(\{y\})=\bar{R}_{\beta}(\{z\})$. Therefore $\bar{R}_{\beta}(\{x\})=\bar{R}_{\beta}(\{y\})=\bar{R}_{\beta}(\{z\})$. Hence, either $\bar{R}_{\beta}(\{x\})=\bar{R}_{\beta}(\{y\})$ or $\bar{R}_{\beta}(\{x\}) \cap \bar{R}_{\beta}(\{y\})=\phi$.

\section{Properties of NeAR Open Approximation Spaces}

The purpose of this section is to investigate some properties of near open approximation spaces.

Proposition 5.1. Let $\left(X, R_{\beta}\right)$ be a near open approximation space and $A, B \subseteq X$. Then

(i) $\underline{R}_{\beta}(A) \subseteq A \subseteq \bar{R}_{\beta}(A)$

(ii) $\underline{R}_{\beta}(\phi)=\bar{R}_{\beta}(\phi)=\phi, \underline{R}_{\beta}(X)=\bar{R}_{\beta}(X)=X$.

(iii) If $A \subseteq B$ then $\underline{R}_{\beta}(A) \subseteq \underline{R}_{\beta}(B)$ and

$\bar{R}_{\beta}(A) \subseteq \bar{R}_{\beta}(B)$

Proof. (i) Let $x \in \underline{R}_{\beta}(A)$ which mean that 
$x \in \bigcup\{G \in \beta O(X), G \subseteq A\} \quad$. Then there exists $G_{0} \in \beta O(X)$ such that $x \in G_{0} \subseteq A$. Thus $x \in A$. Hence $\underline{R}_{\beta}(A) \subseteq A$. Also, let $x \in X$ and by definition of $\bar{R}_{\beta}(A)=\bigcap\{F \in \beta C(X), A \subseteq F\}$, then $x \in F$ for all $F \in \beta C(X)$. Hence $A \subseteq \bar{R}_{\beta}(A)$.

(ii) Follows directly.

(iii) Let $x \in \underline{R}_{\beta}(A)$, by definition of near open lower approximation of $A$, we have $x \in \bigcup\{G \in \beta O(X), G \subseteq A\}$ but $A \subseteq B$, thus $G \subseteq B$ and $x \in G$, then $x \in \underline{R}_{\beta}(B)$. Also, let $x \notin \bar{R}_{\beta}(B)$ this means that $x \notin \bigcap\{F \in \beta C(X), B \subseteq F\}$ then, there exists $F \in \beta C(X), B \subseteq F$ and $x \notin F$ which means that, there exists $F \in \beta C(X), A \subseteq B \subseteq F$ and $x \notin F$ which implies $x \notin \bigcap\{F \in \beta C(X), A \subseteq F\}$, thus $x \notin \bar{R}_{\beta}(A)$. Therefore $\bar{R}_{\beta}(A) \subseteq \bar{R}_{\beta}(B)$.

Proposition 5.2. Let $\left(X, R_{\beta}\right)$ be a near open approximation space and $A, B \subseteq X$. Then

(i) $\underline{R}_{\beta}(X \backslash A)=X \backslash \bar{R}_{\beta}(A)$

(ii) $\bar{R}_{\beta}(X \backslash A)=X \backslash \underline{R}_{\beta}(A)$

(iii) $\underline{R}_{\beta}\left(\underline{R}_{\beta}(A)\right)=\underline{R}_{\beta}(A)$

(iv) $\bar{R}_{\beta}\left(\bar{R}_{\beta}(A)\right)=\bar{R}_{\beta}(A)$

(v) $\underline{R}_{\beta}\left(\underline{R}_{\beta}(A)\right) \subseteq \bar{R}_{\beta}\left(\underline{R}_{\beta}(A)\right)$

(vi) $\underline{R}_{\beta}\left(\bar{R}_{\beta}(A)\right) \subseteq \bar{R}_{\beta}\left(\bar{R}_{\beta}(A)\right)$

Proof. (i) Let $x \in \underline{R}_{\beta}(X \backslash A)$ which is equivalent to $x \in \bigcup\{G \in \beta O(X), G \subseteq X \backslash A\}$. So there exists $G_{0} \in \beta O(X)$ such that $x \in G_{0} \subseteq X \backslash A$. Then there exists $G_{0}^{c}$ such that $A \subset G_{0}^{c}$ and $x \notin G_{0}^{c}, G_{0}^{c} \in \beta C(X)$. Thus, $x \notin \bar{R}_{\beta}(A)$. So $x \in X \backslash \bar{R}_{\beta}(A)$. Therefore $\underline{R}_{\beta}(X \backslash A)=X \backslash \bar{R}_{\beta}(A)$.

(ii) Similar to (i).

(iii) Since $\underline{R}_{\beta}(A)=\bigcup\{G \in \beta O(X), G \subseteq A\}$. This implies that

$$
\begin{aligned}
& \underline{R}_{\beta}\left(\underline{R}_{\beta}(A)\right)=\bigcup\{\bigcup\{G \in \beta O(X), G \subseteq A\}\} \\
& =\bigcup\{G \in \beta O(X), G \subseteq A\}=\underline{R}_{\beta}(A)
\end{aligned}
$$

(iv)

$$
\begin{aligned}
& \bar{R}_{\beta}\left(\bar{R}_{\beta}(A)\right)=\bar{R}_{\beta}\left(X \backslash \underline{R}_{\beta}(X \backslash A)\right)= \\
& X \backslash \underline{R}_{\beta}\left(X \backslash \underline{R}_{\beta}(X \backslash A)\right)
\end{aligned}
$$

and (iii), we get

$\bar{R}_{\beta}\left(\bar{R}_{\beta}(A)\right)=X \backslash \underline{R}_{\beta}(X \backslash A)=X \backslash\left(X \backslash \bar{R}_{\beta}(A)\right)=\bar{R}_{\beta}(A)$

(v) Since $\underline{R}_{\beta}(A) \subseteq \bar{R}_{\beta}\left(\underline{R}_{\beta}(A)\right)$ and by (iii) we have

$\underline{R}_{\beta}\left(\underline{R}_{\beta}(A)\right)=\underline{R}_{\beta}(A)$, then

$\underline{R}_{\beta}\left(\underline{R}_{\beta}(A)\right) \subseteq \bar{R}_{\beta}\left(\underline{R}_{\beta}(A)\right)$.

(vi) Since $\underline{R}_{\beta}\left(\bar{R}_{\beta}(A)\right) \subseteq \bar{R}_{\beta}(A)$ and by (iv), we have

$\bar{R}_{\beta}\left(\bar{R}_{\beta}(A)\right)=\bar{R}_{\beta}(A)$, then

$\underline{R}_{\beta}\left(\bar{R}_{\beta}(A)\right) \subseteq \bar{R}_{\beta}\left(\bar{R}_{\beta}(A)\right)$.

Proposition 5.3. Let $\left(X, R_{\beta}\right)$ be a near open approximation space and $A, B \subseteq X$. Then

(i) $\underline{R}_{\beta}(A \cup B) \supseteq \underline{R}_{\beta}(A) \cup \underline{R}_{\beta}(B)$

(ii) $\bar{R}_{\beta}(A \cup B) \supseteq \bar{R}_{\beta}(A) \cup \bar{R}_{\beta}(B)$

(iii) $\underline{R}_{\beta}(A \cap B) \subseteq \underline{R}_{\beta}(A) \cap \underline{R}_{\beta}(B)$

(iv) $\bar{R}_{\beta}(A \cap B) \subseteq \bar{R}_{\beta}(A) \cap \bar{R}_{\beta}(B)$

Proof. (i) Since we have $A \subseteq A \cup B$ and $B \subseteq A \cup B$. Then $\underline{R}_{\beta}(A) \subseteq \underline{R}_{\beta}(A \cup B)$ and $\underline{R}_{\beta}(B) \subseteq \underline{R}_{\beta}(A \cup B)$ by (iii) in Proposition 5.1, then $\underline{R}_{\beta}(A \cup B) \supseteq \underline{R}_{\beta}(A) \cup \underline{R}_{\beta}(B)$.

(ii), (iii) and (iv) Similar to (i).

The equality of all parts in Proposition 5.3 are not held as shown in the following example.

Example 5.1. According to Example 3.1:

(i) If $A=\{d\}, B=\{a, b\}$, then we have $\underline{R}_{\beta}(A \cup B)=\{a, b, d\}, \underline{R}_{\beta}(A)=\{d\}, \underline{R}_{\beta}(B)=\{a\}$. Therefore $\underline{R}_{\beta}(A \cup B) \neq \underline{R}_{\beta}(A) \cup \underline{R}_{\beta}(B)$.

(ii) If $A=\{d\}, B=\{a, b\}$, then we have $\bar{R}_{\beta}(A \cup B)=X, \bar{R}_{\beta}(A)=\{b, d\}, \bar{R}_{\beta}(B)=\{a, b\}$. Therefore $\bar{R}_{\beta}(A) \cup \bar{R}_{\beta}(B) \neq \bar{R}_{\beta}(A \cup B)$.

(iii) If $A=\{a, b, c\}, B=\{b, c, d\}$, then we have $\underline{R}_{\beta}(A \cap B)=\phi \quad, \quad \underline{R}_{\beta}(A)=\{a, c\} \quad$ and $\underline{R}_{\beta}(B)=\{b, c, d\}$ Therefore $\underline{R}_{\beta}(A \cap B) \neq \underline{R}_{\beta}(A) \cap \underline{R}_{\beta}(B)$.

(iv) If $A=\{b\}, B=\{c, d\}$, then we have 
$\bar{R}_{\beta}(A \cap B)=\phi, \bar{R}_{\beta}(A)=\{b\}, \bar{R}_{\beta}(B)=\{b, c, d\}$.

Therefore $\bar{R}_{\beta}(A) \cap \bar{R}_{\beta}(B) \neq \bar{R}_{\beta}(A \cap B)$. 5.3.

The following theorems are a generalization of Proposition

Theorem 5.1. Let $\left(X, R_{\beta}\right)$ be a near open approximation space and $A, B \subseteq X$. If $A$ is $R_{\beta}$-definable. Then the following are held.

(i) $\underline{R}_{\beta}(A \cup B)=\underline{R}_{\beta}(A) \cup \underline{R}_{\beta}(B)$

(ii) $\bar{R}_{\beta}(A \cap B)=\bar{R}_{\beta}(A) \cap \bar{R}_{\beta}(B)$

Proof. (i) It is clear that $\underline{R}_{\beta}(A) \cup \underline{R}_{\beta}(B) \subseteq \underline{R}_{\beta}(A \cup B)$.

For the converse inclusion, let $x \in \underline{R}_{\beta}(A \cup B)$, that means, $x \in \bigcup\{G \in \beta O(X), G \subseteq A \cup B\}$. Then there exists $G_{0} \in \beta O(X)$ such that $x \in G_{0} \subset A \cup B$. We distinguish three cases:

Case (1) If $G_{0} \subset A, x \in G_{0}$ and $G_{0}$ is a $\beta$-open set, then $x \in \underline{R}_{\beta}(A)$.

Case (2) If $G_{0} \cap A=\phi$, then $G_{0} \subseteq B$ and $x \in G_{0}$, thus $x \in \underline{R}_{\beta}(B)$.

Case (3) If $G_{0} \cap A \neq \phi$. Since $x \in G_{0}$ and $G_{0}$ is a $\beta$ open set, then $x \in \beta \operatorname{cl}(A)$, for every $G_{0}$ which has the above condition, thus, thus $x \in \bar{R}_{\beta}(A)$, then $x \in \underline{R}_{\beta}(A)$, because $A$ is $R_{\beta}$ - definable. Hence, in three cases $x \in \underline{R}_{\beta}(A) \cup \underline{R}_{\beta}(B)$.

(ii) It is clear that $\bar{R}_{\beta}(A \cap B) \subseteq \bar{R}_{\beta}(A) \cap \bar{R}_{\beta}(B)$. We prove the converse inclusion. Let $x \in \bar{R}_{\beta}(A) \cap \bar{R}_{\beta}(B)$, then $x \in \bar{R}_{\beta}(A)$ implies $x \in \underline{R}_{\beta}(A)$ and $x \in G \subseteq X$, where $G$ is a $\beta$-open set and $x \in \bar{R}_{\beta}(B)$ implies for all $G \in \beta O(X) \quad, \quad G \cap B \neq \phi \quad$. Therefore $G \cap(A \cap B)=(G \cap A) \cap B=G \cap Y \neq \phi$. Hence $x \in \bar{R}_{\beta}(A \cap B)$.

Theorem 5.2. Let $\left(X, R_{\beta}\right)$ be a near open approximation space and $A, B \subseteq X$. Then the following are held.

(i) $\bar{R}_{\beta}(\operatorname{cl}(A) \cup B)=\operatorname{cl}(A) \cup \bar{R}_{\beta}(B)$

(ii) $\underline{R}_{\beta}(\operatorname{int}(A) \cap B)=\operatorname{int}(A) \cap \underline{R}_{\beta}(B)$

Proof. (i) By Proposition 5.1 (i) and Proposition 5.3 (ii), we have

$$
\operatorname{cl}(A) \subset \bar{R}_{\beta}(\operatorname{cl}(A))
$$

Then $c l(A) \cup \bar{R}_{\beta}(B) \subset \bar{R}_{\beta}(c l(A)) \cup \bar{R}_{\beta}(B)$ $\subset \bar{R}_{\beta}(\operatorname{cl}(A) \cup B)$

- On the other hand, since $\operatorname{cl}(A) \cup B \subset \operatorname{cl}(A) \cup \bar{R}_{\beta}(B)$ and the union of a $\beta$-open set and a closed set is $\beta$-closed, then $\bar{R}_{\beta}(\operatorname{cl}(A) \cup B) \subset \bar{R}_{\beta}\left(\operatorname{cl}(A) \cup \bar{R}_{\beta}(B)\right)=\operatorname{cl}(A) \cup \bar{R}_{\beta}(B)$ . Therefore, $\bar{R}_{\beta}(\operatorname{cl}(A) \cup B)=\operatorname{cl}(A) \cup \bar{R}_{\beta}(B)$.

(ii) Since the intersection of an open set $\operatorname{int}(A)$ and a $\beta$ open set $\underline{R}_{\beta}(B) \quad$ is $\quad \beta \quad$-open, $\operatorname{int}(A) \cap \underline{R}_{\beta}(B)=\underline{R}_{\beta}\left(\operatorname{int}(A) \cap \underline{R}_{\beta}(B)\right) \subset \underline{R}_{\beta}(\operatorname{int}(A) \cap B)$ . On the other hand, by using Proposition 5.3 (iii), $\underline{R}_{\beta}(\operatorname{int}(A) \cap B) \subset \underline{R}_{\beta}(\operatorname{int}(A)) \cap \underline{R}_{\beta}(B) \subset \operatorname{int}(A) \cap \underline{R}_{\beta}(B)$ . Therefore $\underline{R}_{\beta}(\operatorname{int}(A) \cap B)=\operatorname{int}(A) \cap \underline{R}_{\beta}(B)$.

\section{CONCLUSIONS}

In this paper, we used general binary relations to generate rough approximations. Properties of rough belonging, rough equality and rough power set are now clear with respect to any relation type raised from any field of applications.

We have proved that there exist similar properties and there exist different properties among the approximations raised from this paper and that studied from many authors $[5,6,7,11,13,14,15,16,25,27,30]$. We will investigate new applications of these tools using topological generalizations in a future paper.

\section{REFERENCES}

[1] H. M. Abu-Donia, "Multi knowledge based rough approximations and applications", Knowledge-Based Systems 26 (2012) 20-29.

[2] H. M. Abu-Donia, A. S. Salama, "Generalization of Pawlak's rough approximation spaces by using $\delta \beta$-open sets", International Journal of Approximate Reasoning 53 (2012) 1094-1105.

[3] D. G. Chen, W. X. Zhang, "Rough sets and topological spaces", Journal of Xian Jiaotong University 35 (2001) 1313-1315.

[4] A. Galton, "A generalized topological view of motion in discrete space", Theoretical Computer Science 305 (1-3) (2003) 111-134.

[5] J. L. Kelley, "General Topology", Van Nostrand Company, 1995.

[6] M. Kondo, "On the structure of generalized rough sets", Information Sciences 176 (2006) 589-600.

[7] J. Kortelainen, "On the relationship between modified sets, topological spaces and rough sets", Fuzzy Sets and Systems 61(1994) 91-95.

[8] M. Kryszkiewicz, "Rough set approach to incomplete information systems", Information Sciences 112 (1998) 39-49.

[9] M. Kryszkiewicz, "Rules in incomplete information systems", Information Sciences 113 (1999) 271-292.

[10] E. F. Lashin, A. M. Kozae, A. A. Abo Khadra, T. Medhat, "Rough set theory for topological spaces", International Journal of Approximate Reasoning 40 (2005) 35-43.

[11] C. Largeron, S. Bonnevay, "A pre topological approach for structure analysis", Information Sciences 144 (2002) 185-196.

[12] Zhaowen Li, Tusheng Xie, Qingguo Li, "Topological a structure of generalized rough sets", Computers and Mathematics with Applications 63 (6) (2012) 1066-1071.

[13] T. Y. Lin, "Granular fuzzy sets: A view from rough set and probability theories", International Journal of Fuzzy Systems 3 (2) (2001) 373-381. 
[14] Guilong Liu, Kai Zhu, "The relationship among 3 types of rough approximation pairs", Knowledge-Based Systems 60 (2014) 28-34.

[15] Zhi Pei, Daowu Pei and Li Zheng, "Topology vs generalized rough sets", International Journal of Approximate Reasoning 58 (2012) 221249.

[16] Z. Pawlak, "Rough sets", International Journal of Computer and Information Sciences 11 (5) (1982) 341-356.

[17] Z. Pawlak, "Rough sets and fuzzy sets", Fuzzy Set and Systems 17
(1985) 99-102.

[18] K. Y. Qin, Z. Pei, "On the topological properties of fuzzy rough sets", Fuzzy Sets and Systems 151 (3) (2005) 601-613.

[19] K. Y. Qin, J. L. Yang, Z. Pei, "Generalized rough sets based on reflexive and transitive relations", Information Sciences 178 (2008) 4138-4141.

[20] A. S. Salama, "Topological solution of missing attribute values problem in incomplete information tables", Information Sciences 180 (2010) 631639. 